\title{
Griginal Evaluation of acoustic radiation force impulse elastography (ARFI) in the diagnosis of hepatic fibrosis and portal hypertension
}

\author{
DN Amarapurkar, R Kashikar, S Lala, S Gautam, \\ AD Amarapurkar
}

ABSTRACT

Department of gastroenterology \& Hepatology, Bombay Hospital \& medical research centre and SRL \& Dr Avinash Phadke Laboratories, Mumbai, India.

Correspondence: Deepak N Amarapurkar Email: amarapurkar@gmail.com

Background: Transient elastography by fibroscan is widely used as non-invasive marker for liver fibrosis. Fibroscan has limited accuracy in patients with elevated serum bilirubin, ALT, ascites, BMI $>30 \mathrm{~kg} / \mathrm{m}^{2}$ and requires separate equipment for evaluation. ARFI is a new way of assessing liver fibrosis which is incorporated in ultrasound machine. In a prospective study we evaluated utility of ARFI in 171 consecutive patients undergoing liver biopsy and assessed misclassification rate of fibrosis in patients with elevated serum bilirubin, ALT, BMI $>25 \mathrm{~kg} / \mathrm{m}^{2}$ and ascites.

Methods: 171 consecutive patients (mean age $46.04 \pm 13.34$ years, males $60 \%$ ) undergoing liver biopsy were evaluated for ARFI by Acuson S2000, Siemens ultrasonography machine. Indications for liver biopsy were autoimmune liver disease (38), alcoholic liver disease (13), non-alcoholic fatty liver disease (24), cryptogenic liver disease (10), noncirrhotic portal hypertension (29), viral hepatitis (17) and miscellaneous (40). Liver biopsy was graded using Metavir classification. Aspartate aminotransferase to platelet ratio index (APRI) was calculated in each patient. ARFI and APRI values were correlated with fibrosis stage on liver biopsy. ARFI and fibrosis were compared with respect to serum bilirubin, ALT, BMI $>25 \mathrm{~kg} / \mathrm{m}^{2}$ and ascites.

Results: Mean ARFI score with interquartile range were $1.70 \pm 0.46$ (1.33-1.99) in stage $0,1.92 \pm 0.56(1.45-2.16)$ in stage $1-2$ and $2.49 \pm 0.60$ (2.07-2.98) in stage 3 and 4 fibrosis. Mean ARFI and APRI values were significantly higher in stage 3 and 4 fibrosis as compared to fibrosis stage 0 and 1-2 (p 0.001). ARFI values were misclassified in patients with bilirubin $>10 \mathrm{mg} / \mathrm{dl}$, ALT > $300 \mathrm{IU} / \mathrm{L}$ and ascites.

Conclusion: ARFI was reliable predictor of advanced fibrosis but could not differentiate stage 0 from early fibrosis. ARFI values were not reliable in bilirubin $>10 \mathrm{mg} / \mathrm{dl}$, ALT $>300 \mathrm{IU} / \mathrm{L}$ and ascites.

KEYWORDS: NAFLD; Liver stiffness; liver biopsy; misclassification rate. 


\section{Introduction}

Liver fibrosis is a consequence of chronic inflammation which leads to accumulation of extracellular matrix including collagen in the space of Disse. This occurs in majority of chronic liver diseases such as hepatitis $\mathrm{B}$, hepatitis $\mathrm{C}$ infection, alcoholic liver disease, nonalcoholic fatty liver disease (NAFLD), drugs or autoimmune liver diseases. Though the etiological agents differ, the pathophysiological responses are similar leading to fibrosis and cirrhosis. Cirrhosis is the end stage of fibrosis, which is characterised by the loss of normal architecture and vascular remodelling giving rise to portal hypertension. ${ }^{1,2}$ As the prognosis of chronic liver disease depends on the extent of fibrosis, recognition of fibrosis at earlier stages is preferable, as timely and appropriate treatment can reverse or prevent the cirrhosis.

Liver biopsy is considered the 'gold standard' for assessment of liver fibrosis. ${ }^{3}$ However it is an invasive procedure having several limitations in the form of complications, sampling errors and interobserver variability. ${ }^{4,5}$ Recently a large number of non-invasive tests for hepatic fibrosis are available. ${ }^{6,7}$ They are in the form of either serum markers (direct or indirect) or imaging modalities. ${ }^{8-17}$ Serum markers have major limitations to identify early stages of fibrosis.

Liver imaging techniques which measure liver stiffness that correlates with liver fibrosis using ultrasound waves such as: Transient elastography (TE or Fibroscan), Sono elastography (Real-Time Tissue elastography), Acoustic Radiation Force Impulse (ARFI), Shear Wave elastography and Super Sonic Shear Imaging., ${ }^{71-21}$ These ultrasound elastography methods have advantage over serologic methods regarding the fact that the readings can be obtained immediately without waiting for laboratory results. TE is based on ultrasound technology, which correlates liver fibrosis with hepatic elasticity. TE has limited accuracy in elevated bilirubin, ALT, ascites and with sever steatosis. ARFI elastography is a new way of assessing liver fibrosis which is incorporated in ultrasound machine. ARFI is less influenced by steatosis and has been shown to be useful in patients with ascites. Hence the aim of this study was to evaluate ARFI in patients undergoing liver biopsy and to assess misclassification rate of fibrosis in patients with elevated serum bilirubin, ALT, BMI $>25$ $\mathrm{kg} / \mathrm{m}^{2}$ and ascites.

\section{Material and Methods}

In this prospective observational study we included 171 patients (mean age $46.04 \pm 13.34$ years, males $60 \%$ ) undergoing liver biopsy during November 2012 to April 2014. Indications for liver biopsy were, autoimmune liver disease 38 (22.2\%), alcoholic liver disease 13 (7.6\%), non-alcoholic fatty liver disease 24 (14\%), cryptogenic liver disease 10 (5.8\%), noncirrhotic portal hypertension 29 (16.9\%), viral hepatitis 17 (9.9\%) and miscellaneous conditions 40 (23.3\%) which included granulomatous liver disease, drug induced liver disease (DILI) and cholestatic liver disease. Patients were evaluated with appropriate clinical history, physical examination, laboratory evaluation and endoscopy. Physical parameters such as height (meters), weight $(\mathrm{Kg})$ and BMI $\left(\mathrm{Kg} / \mathrm{m}^{2}\right)$ were recoded. Patients were subjected to complete blood count (hemoglobin, mean corpuscular volume, WBC and platelet count), liver function tests (bilirubin total and direct, AST, ALT, ALP, GGT, total protein, albumin and INR), viral and autoimmune markers. Aspartate aminotransferase to platelet ratio (APRI index) was calculated in each patient. Diagnosis of autoimmune liver diseases was done using standard international guidelines. ${ }^{22}$ Ultrasonography evaluation of abdomen was done to determine liver and spleen size, echo texture, ascites and portal vein size. Doppler evaluation of portal and hepatic venous system was done in cases of chronic liver disease, suspected non cirrhotic portal hypertension $(\mathrm{NCPH})$ and extrahepatic portal vein obstruction (EHPVO). Patients were subjected to upper gastrointestinal endoscopy. Esophageal varices were graded using Japanese Research Society criteria as no varices, small and large varices. ${ }^{23}$ This study was approved by Institutional ethics committee.

ARFI was performed (Acuson S2000, Siemens) to evaluate liver and splenic stiffness in all patients. ARFI was done on empty stomach. ARFI scanning was done between the ribs in the right liver lobe with minimal scanning pressure applied by the operator, patient lying 
in the dorsal decubitus position and momentary breath holding in left lobe of liver. A measurement depth of 2-3 $\mathrm{cm}$ below the liver capsule is chosen to standardize the examination. Similarly, spleen measurements for stiffness was taken in intercostal spaces, $1 \mathrm{~cm}$ below the spleen capsule with patient laying in right lateral decubitus and arm maximally abducted. Ten acquisitions of ARFI were performed for each patient and mean value is calculated for reliable measurement for liver and spleen stiffness. Sixty one individuals, age and sex matched without liver disease were taken as controls.

Percutaneous liver biopsy was done under ultrasound guidance on the same day of ARFI. Liver biopsy specimens were processed routinely. Special stain, Masson's trichrome was done for assessment of fibrosis. Biopsy specimen with more than 10 portal tracts were said to be adequate and examined by experienced hepatopathologist. Biopsies were assessed by Histologic Activity Index (HAI). METAVIR scoring system was used for staging of fibrosis. $\mathrm{F} 0=$ no fibrosis. F1=periportal fibrosis. F2=Fibrous septa going away from portal areas. $\mathrm{F} 3=$ bridging fibrosis. $\mathrm{F} 4=$ cirrhosis. ${ }^{24}$ Steatosis (macrovesicular) was graded according to the non-alcoholic fatty liver disease activity score as follows: Grade $0:<5 \%$ of hepatocytes showing macrovesicular steatosis. Grade 1: 5\%-33\%.Grade 2:>33\%-66\% and Grade 3: $>66 \%$ steatosis. ${ }^{25}$

ARFI and APRI values were correlated with fibrosis stage on liver biopsy. ARFI and fibrosis were compared with respect to serum bilirubin, ALT, BMI, ascites, steatosis and esophageal varices. Statistical analysis was done using SPSS version 17 software. Quantitative data was presented as mean and standard deviation and analysed using student's t -test. Qualitative data was presented as frequency and percentage and analysed using chi-square test. $\mathrm{P}$ value of $<0.05$ was considered as significant.

\section{Results}

Control population showed mean ARFI of $1.41 \pm 0.26 \mathrm{~m} / \mathrm{s}$. Mean ARFI score and interquartile range were $1.70 \pm 0.46$ (1.33-1.99) in stage 0 fibrosis, $1.92 \pm 0.56(1.45-2.16)$ in stage 1-2 fibrosis and 2.49 $\pm 0.60(2.07-2.98)$ in stage 3-4 fibrosis respectively. Mean ARFI was significantly higher $(p=0.001)$ in stage 3-4 fibrosis with 95\% confidence interval of 1.31-3.67. There was no statistical difference $(p=0.77)$ in ARFI score between stage 0 and 1-2 fibrosis. (Table1).

ARFI values and liver fibrosis were compared in patients with total bilirubin $>10 \mathrm{mg} / \mathrm{dl}$ and $\leq 10 \mathrm{mg} / \mathrm{dl}$. In stage 0 fibrosis ,mean ARFI was $2.17 \pm 0.58$ with total bilirubin $>10 \mathrm{mg} / \mathrm{dl}$ which was significantly higher $(\mathrm{p}=0.002)$ as compared to $1.59 \pm 0.34$ with total bilirubin $\leq 10 \mathrm{mg}$. For stages 1 to 4 fibrosis, mean ARFI values were high in patients with total bilirubin $>10 \mathrm{mg} / \mathrm{dl}$ as compared to $\leq 10 \mathrm{mg} / \mathrm{dl}$. ARFI was miscalculated in all stages of fibrosis with bilirubin $>10 \mathrm{mg} / \mathrm{dl}$. (Table 2).

ARFI overestimated stages of fibrosis in patients with ALT $>300 \mathrm{IU} / \mathrm{L}$. Mean ARFI for stage 0 and 2 fibrosis was $2.48 \pm 0.51$ and $2.46 \pm 0.0$ with ALT $>300$ $\mathrm{IU} / \mathrm{L}$ and $1.66 \pm 0.42$ and $1.77 \pm 0.46$ with $\mathrm{ALT} \leq 300$

Table 1: Association between ARFI score and fibrosis stage

\begin{tabular}{|c|c|c|c|c|}
\hline $\begin{array}{c}\text { Fibrosis } \\
\text { stage }\end{array}$ & $\begin{array}{l}\text { Mean } \\
\text { ARFI } \\
\text { score } \\
( \pm \text { SD) }\end{array}$ & $\begin{array}{l}\text { Median } \\
\text { (Inter } \\
\text { quartile } \\
\text { range } \\
\text { Q1-Q3) }\end{array}$ & $95 \% \mathrm{CI}$ & P value \\
\hline $\begin{array}{c}0 \\
(\mathrm{~N}=54)\end{array}$ & $1.70 \pm 0.46$ & $\begin{array}{c}1.59 \\
(1.33-1.99) \\
\end{array}$ & $0.80-2.50$ & \\
\hline $\begin{array}{c}1-2 \\
(\mathrm{~N}=28) \\
\end{array}$ & $1.92 \pm 0.56$ & $\begin{array}{c}1.91 \\
(1.45-2.16) \\
\end{array}$ & $0.82-3.02$ & $\begin{array}{c}0.077 \\
\text { NS }\end{array}$ \\
\hline $\begin{array}{c}3-4 \\
(\mathrm{~N}=87)\end{array}$ & $2.49 \pm 0.60$ & $\begin{array}{c}2.45 \\
(2.07-2.98)\end{array}$ & $1.31-3.67$ & $* 0.001$ \\
\hline
\end{tabular}

${ }^{*}$ v value $=$ significant

Table 2: ARFI values with fibrosis and total bilirubin

\begin{tabular}{c|c|c|c|c}
\multirow{2}{*}{$\begin{array}{c}\text { Fibrosis } \\
\text { stage }\end{array}$} & \multicolumn{4}{|c}{ Mean ARFI values ( $\mathbf{N}$ SD) } \\
\cline { 2 - 5 } & $\begin{array}{c}\text { T. Bilirubin } \\
(>\mathbf{1 0} \mathbf{m g} / \mathbf{d l})\end{array}$ & $\mathbf{N}$ & $\begin{array}{c}\text { T. Bilirubin } \\
(\leq \mathbf{1 0 m g} / \mathbf{d l})\end{array}$ \\
\hline 0 & 13 & $* 2.17 \pm 0.58$ & 43 & $1.59 \pm 0.34$ \\
\hline 1 & 2 & $2.90 \pm 0.52$ & 11 & $1.89 \pm 0.53$ \\
\hline 2 & 1 & $2.51 \pm 0.00$ & 14 & $1.77 \pm 0.45$ \\
\hline 3 & 5 & $2.61 \pm 0.57$ & 31 & $2.32 \pm 0.56$ \\
\hline 4 & 2 & $2.74 \pm 0.48$ & 49 & $2.57 \pm 0.62$
\end{tabular}

*p value=significant 
IU/L respectively and this difference was statistically significant. $(p<0.05)$. (Table 3).

ARFI value in patients with BMI $>25 \mathrm{~kg} / \mathrm{m} 2$ and $\leq 25 \mathrm{~kg} / \mathrm{m}^{2}$ was compared. No significant difference was found in ARFI with BMI $>25 \mathrm{~kg} / \mathrm{m}^{2}$ or $\leq 25 \mathrm{~kg} / \mathrm{m}^{2}$. ARFI values were not affected in patients with high BMI.

Mean ARFI in patients with ascites was higher in all stages of fibrosis as compared to patients without ascites. However the difference was not statistically significant.

ARFI was evaluated in patients with less than and more than grade 1 steatosis on liver biopsy. Mean ARFI at stage 0 fibrosis was $1.27 \pm 0.11$ in patients with $>$ grade 1 steatosis and $1.75 \pm 0.46$ with $<$ grade 1 steatosis. This difference was statistically significant. $(p=0.001)$. In remaining stages of fibrosis there was no difference in ARFI with respect to steatosis.

Liver and spleen ARFI was measured in patients with small, large esophageal varices and without varices. Liver ARFI was $2.06 \pm 0.6$ in patients without varices, $2.3 \pm 0.7$ with small and $2.2 \pm 0.6$ large varices. While splenic ARFI was $2.7 \pm 0.5$ without varices, $3.2 \pm 0.5$ with small and $3.2 \pm 0.3$ large varices. ARFI values of the liver did not correlate with presence and size of esophageal varices. Splenic ARFI values were significantly higher as compared to liver ARFI $(\mathrm{p}<0.05)$ and it could differentiate between presence and absence of varices.

Aspartate aminotransferase to platelet ratio (APRI index) was compared with fibrosis stage on liver biopsy. Mean APRI (1.95 \pm 4.10$)$ was significantly high in stage 3-4 fibrosis with 95\% confidence interval of 6.09-9.99. APRI could not differentiate between stage 0 and stage 1-2 fibrosis.

Mean ARFI in control population was found to be $1.41 \pm 0.26 \mathrm{~m} / \mathrm{s}$. Mean ARFI in patients with non-cirrhotic portal hypertension $(\mathrm{NCPH})$ without fibrosis was higher $(1.60 \pm 0.29)$ than control population but lesser than chronic liver disease patients $(1.70 \pm 0.46)$. However the difference was not statistically significant.

When ARFI was evaluated in patients with ALT $<300 \mathrm{IU} / \mathrm{L}$, total bilirubin $<10 \mathrm{mg} / \mathrm{dl}$, and without ascites, mean ARFI (2.50 \pm 0.59$)$ correlated significantly ( $\mathrm{p}=0.001)$ with stage 3-4 fibrosis. In these patients mean ARFI for stage 1-2 was higher $(1.76 \pm 0.40)$ than stage 0 fibrosis $(1.58 \pm 0.36)$. However this difference was not statistically significant. $(\mathrm{p}=0.170)$ (Table 4).

In this study ARFI was misclassified in overall $36.2 \%$ of cases. These were $21(12.3 \%)$ of patients with total bilirubin $>10 \mathrm{mg} / \mathrm{dl}, 9(5.3 \%)$ ALT $>300 \mathrm{IU} / \mathrm{L}$, 32(18.7\%) with ascites. (Table 5).

\section{Discussion}

TE, the most widely used method for assessment of liver stiffness has major limitations in patients with

Table 3: Association between ARFI values with fibrosis and ALT

\begin{tabular}{c|c|c|c|c}
\multirow{2}{*}{$\begin{array}{c}\text { Fibrosis } \\
\text { stage }\end{array}$} & \multicolumn{4}{|c}{ Mean ARFI values ( $\mathbf{N}$ SD) } \\
\cline { 2 - 5 } & $\mathbf{N}$ & $\begin{array}{c}\text { ALT } \\
(>\mathbf{3 0 0} \text { IU/L) }\end{array}$ & $\mathbf{N}$ & $\begin{array}{c}\text { ALT } \\
(\leq \mathbf{3 0 0} \text { IU/L) }\end{array}$ \\
\hline 0 & 3 & $2.48 \pm 0.51$ & 51 & $1.66 \pm 0.42$ \\
\hline 1 & 0 & - & 13 & $2.04 \pm 0.64$ \\
\hline 2 & 1 & $2.46 \pm 0.00$ & 14 & $1.77 \pm 0.46$ \\
\hline 3 & 3 & $2.09 \pm 0.73$ & 33 & $2.38 \pm 0.56$ \\
\hline 4 & 2 & $2.39 \pm 0.01$ & 49 & $2.58 \pm 0.62$
\end{tabular}

${ }^{*} p<0.05$

Table 4: ARFI in patients with ALT $<300$ IU/L, Bilirubin < $10 \mathrm{mg} / \mathrm{dl}$ and without ascites

\begin{tabular}{c|c|c} 
Fibrosis stage & $\begin{array}{c}\text { Mean ARFI } \\
\text { values ( } \pm \text { SD })\end{array}$ & P value \\
$\begin{array}{c}0 \\
(\mathrm{~N}=25)\end{array}$ & $1.58 \pm 0.36$ & - \\
\hline $\begin{array}{c}1-2 \\
(\mathrm{~N}=14)\end{array}$ & $1.76 \pm 0.40$ & $\begin{array}{c}0.17 \\
\mathrm{NS}\end{array}$ \\
\hline $\begin{array}{c}3-4 \\
(\mathrm{~N}=42)\end{array}$ & $2.50 \pm 0.59$ & $* 0.001$
\end{tabular}

*p value $=$ significant

Table 5: Missed classification rate

\begin{tabular}{c|c|c} 
Parameter & $\begin{array}{c}\text { No. of cases } \\
(\mathbf{N}=\mathbf{1 7 1})\end{array}$ & $\begin{array}{c}\text { Percentage of } \\
\text { cases }\end{array}$ \\
\hline Bilirubin $(>10 \mathrm{mg} / \mathrm{dl})$ & 21 & 12.3 \\
\hline ALT $(>300 \mathrm{IU} / \mathrm{L})$ & 09 & 5.3 \\
\hline Ascites $($ Present $)$ & 32 & 18.7 \\
\hline Either One & 62 & $36.2 \%$
\end{tabular}


overweight, obesity and ascites. ${ }^{26,27}$ Over last three years, several studies have shown the usefulness of ARFI elastography for the assessment of liver stiffness. ${ }^{28-32}$ Results demonstrated that shear-wave velocity measured by ARFI elastography offers a reliable and non-invasive method for estimating liver fibrosis, even in presence of steatosis and ascites. Majority of studies on ARFI are limited to only one liver disease such as hepatitis B, C infection or NAFLD. ${ }^{20,21,30,33}$ There is limited data of ARFI in patients with other liver diseases. In this study we have analysed role of ARFI in various liver diseases such as autoimmune liver disease, alcoholic liver disease, nonalcoholic fatty liver disease, cryptogenic liver disease, noncirrhotic portal hypertension, viral hepatitis and miscellaneous conditions which included granulomatous liver disease, drug induced liver disease (DILI) and cholestatic liver disease. Our results have shown that ARFI had good predictive value for diagnosis of stage 3-4 fibrosis with $95 \%$ confidence interval of 1.31 to 3.67. However it failed to correlate with lesser degree of fibrosis. These observations were similar to Yap et $\mathrm{al}^{34}$ who have assessed feasibility of ARFI in 70 patients of chronic liver disease and have shown strong correlation of ARFI with higher fibrosis scores compared to lower ones. Haque et $\mathrm{al}^{35}$ investigated feasibility of ARFI in 21 patients of chronic liver disease $(\mathrm{HCV}=16, \mathrm{HBV}=1$, Both $\mathrm{HBV} \& \mathrm{HCV}=1$, alcoholic liver disease $=1$, others $=2$ ). Strong correlation was observed between ARFI and fibrosis. ARFI accuracy was maximum in predicting severe fibrosis and cirrhosis. Takahashi et al 36 evaluated ARFI for fibrosis staging in 55 consecutive patients of chronic liver disease. Liver stiffness measured by ARFI correlated with histological fibrosis. $(p<0.0001)$ with highest confidence in stage 4 fibrosis.

TE has been reported to have impaired specificity in presence of cholestasis, obesity, steatosis, elevated bilirubin and ALT. ${ }^{37-42}$ In this study we have observed similar limitations for ARFI. Overall $36.2 \%$ of cases were misclassified as high ARFI. These were patients with high serum bilirubin, raised ALT and presence of ascites. ARFI values could correlate with all stages of fibrosis when serum bilirubin was $<10 \mathrm{mg} / \mathrm{dl}$, ALT $<300 \mathrm{IU} / \mathrm{L}$ and patient without ascites. Pfeifer et $\mathrm{al}^{37}$ have shown that cholestasis significantly increases liver stiffness measured with ARFI. Patients with sonographic cholestasis and increased cholestatic blood values (Bilirubin, $\gamma \mathrm{GT}$, ALP $>2$ times ULN) were included in their study. ARFI values were above the cut-off for cirrhosis $(1.8 \mathrm{~m} / \mathrm{s})$ in all patients with a mean of $2.91 \mathrm{~m} / \mathrm{s} \pm 0.89 \mathrm{~m} / \mathrm{s}$ without a history or signs of cirrhosis. Attia et $\mathrm{al}^{38}$ have shown increased liver stiffness in biliary obstruction which was decreased after endoscopic retrograde cholangiography irrespective of the aetiology. In acute liver failure, hepatic necrosis can mimic liver cirrhosis by ARFI estimation. Karlas et $\mathrm{al}^{39}$ compared ARFI in 3 patients with acute liver failure with healthy controls $(n=33)$ and cases with liver cirrhosis $(n=21)$. ARFI values were significantly increased in acute liver failure as compared to healthy controls $(p=0.001)$ and in patients with cirrhosis. Of 3 patients two underwent liver transplantation. Explants showed massive necrosis, but no signs of chronic liver disease. Third patient recovered spontaneously and showed reduced ARFI results during follow-up.

Kuroda et $\mathrm{al}^{40}$ measured liver stiffness in patients with acute liver failure using ARFI for predicting the prognosis of these patients. ARFI was found to be significantly higher in fulminant hepatitis than in acute hepatitis $(\mathrm{P}<0.001)$.

Hepatic steatosis and obesity are other factors that influence liver stiffness. Fatty tissues are softer and are known to reduce ARFI values..$^{30,41-43}$ In this study AFRI was higher in patients with $>$ grade 1 steatosis at stage 0 fibrosis. In remaining stages of fibrosis there was no difference in ARFI with severity of steatosis. Dong 44 et al studied various non-invasive markers (ARFI, fibroscan and Ferns' index) for fibrosis in chronic hepatitis B patients. They have assessed impact of inflammatory activity and steatosis. Diagnostic accuracy for fibrosis with these methods was good in presence of inflammation $(\mathrm{p}<0.001)$ but not with steatosis $(\mathrm{p}=0.407)$.

Non-invasive methods for diagnosing esophageal varices in cirrhotic patients are required to avoid unnecessary invasive screening endoscopic examinations. TE can predict clinically significant portal hypertension however it has low specificity to determine esophageal varices. In this study liver ARFI could not correlate with size of varices but splenic ARFI could differentiate between presence and absence of varices. Morishita 
et $\mathrm{a}^{45}$ investigated liver stiffness by ARFI for diagnosis of esophageal varices and high-risk esophageal varices among patients with HCV cirrhosis. ARFI value increased significantly is patients with esophageal varices $(p<0.001)$. The ARFI value for high-risk varices was significantly higher than that for low-risk varices $(\mathrm{p}<0.001)$.

Mean ARFI in patients with ascites was higher as compared to those without ascites in this study. Bota and Sporea et $\mathrm{a}^{46}$ have shown that mean liver stiffness measurements by ARFI were statistically and significantly higher in cirrhotic with ascites than in those with noncirrhotic ascites: $3.04 \pm 0.70$ vs $1.45 \pm 0.59 \mathrm{~m} / \mathrm{s}(\mathrm{P}<0.001)$. Salzl et a ${ }^{29}$ evaluated 88 patients with cirrhosis for portal hypertension and esophageal varices using TE and ARFI. TE was not reliable method for liver stiffness in 22(25\%) patients and ARFI in $1(1 \%)$ patient, due to ascites or obesity. Sensitivity and specificity for ARFI (AUC 0.855) was higher ( $71.4 \%$ and $87.5 \%$, respectively) as compared to other methods.

In NCPH, ARFI value was found to be higher than controls and lower than patients with cirrhosis in this study. This indicates that in patients with established portal hypertension, ARFI values less than stage 3-4 fibrosis can predict diagnosis of NCPH. This is similar to study by Sharma et al. ${ }^{47}$ They have evaluated liver and splenic stiffness by TE in 65 patients of extrahepatic portal vein obstruction (EHPVO). Liver and splenic stiffness were found to be higher in EHPVO than controls. Patients with variceal bleed had higher splenic stiffness than those without bleed.

In conclusion, ARFI was reliable predictor of advanced fibrosis. However ARFI could not differentiate between stage 0 and early fibrosis. ARFI values were not reliable in patients with bilirubin $>10 \mathrm{mg} / \mathrm{dl}$, ALT $>300$ $\mathrm{IU} / \mathrm{L}$ and ascites.

\section{References}

1. Wallace K, Burt AD, Wright MC. Liver fibrosis. Biochem J. 2008;411:1-18.

2. Pinzani M, Rosselli M, Zuckermann M. Liver cirrhosis. Best Pract Res Clin Gastroenterol. 2011;25:281-90.

3. Rockey DC, Caldwell SH, Goodman ZD, Nelson RC,
Smith AD; American Association for the Study of Liver Diseases. Liver biopsy. Hepatology. 2009;49:1017-44.

4. Regev A, Berho M, Jeffers LJ et al. Sampling error and intraobserver variation in liver biopsy in patients with chronic HCV infection. Am J Gastroenterol. 2002;97:26148.

5. Bedossa P, Dargère D, Paradis V. Sampling variability of liver fibrosis in chronic hepatitis C. Hepatology. 2003;38:1449-57.

6. Rockey DC, Bissell DM. Noninvasive measures of liver fibrosis. Hepatology. 2006;43:S113-S120.

7. Rockey DC. Non-invasive assessment of liver fibrosis and portal hypertension with transient elastography. Gastroenterology. 2008;134:8-14.

8. Bonacini M, Hadi G, Govindarajan S, Lindsay KL. Utility of adiscriminant score for diagnosing advanced fibrosis or cirrhosis inpatients with chronic hepatitis $\mathrm{C}$ virus infection. Am J Gastroenterol. 1997;92:1302-1304.

9. Forns X, Ampurdanes S, Llovet JM et al. Identification of chronic hepatitis $\mathrm{C}$ patients without hepatic fibrosis by a simple predictive model. Hepatology. 2002;36:986-992.

10. Koda M, Matunaga Y, Kawakami M et al. Fibroindex, a practical index for predicting significantfibrosis in patients with chronic hepatitis C. Hepatology. 2007;45:297-306.

11. Loaeza-del-Castillo A, Paz-Pineda F , Oviedo-Cárdenas E, Sánchez-Ávila F, Vargas-Vorácková . ALT to platelet ratio index (APRI) for the noninvasive evaluation of liver fibrosis. Annals of Hepatology. 2008; 7: 350-357.

12. Imbert-Bismut F, Ratziu V, Pieroni L et al. Biochemical markers of liver fibrosis in patients withhepatitis $\mathrm{C}$ virus infection: a prospective study. Lancet. 2001;357:10691075.

13. Naveau S, Poynard T, Benattar C, Bedossa P, Chaput JC. Alpha 2-macroglobulin and hepatic fibrosis. Diagnostic interest. Dig Dis Sci. 1994;39:2426-2432.

14. Pares A, Deulofeu R, Gimenez A et al. Serum hyaluronate reflects hepatic fibrogenesis in alcoholic liver disease and is useful as a marker of fibrosis. Hepatology. 1996;24:13991403.

15. Suzuki A, Angulo P, Lymp J et al. Hyaluronic acid, an accurate serum marker for severe hepaticfibrosis in patients with non-alcoholic fatty liver disease. Liver Int. 2005;25:779-786.

16. Castera L, Hezode C, Roudot-Thoraval F et al. Worsening of steatosis is an independent factor of fibrosis progression in untreated patients with chronic hepatitis $\mathrm{C}$ and paired liver biopsies. Gut. 2003;52:288-292. 
17. Gordon A, McLean CA, Pedersen JS, Bailey MJ, Roberts SK. Hepatic steatosis in chronic hepatitis B and C: predictors, distribution and effect on fibrosis. $J$ Hepatol. 2005;43:38-44.

18. Friedrich-Rust $\mathrm{M}$, Wunder $\mathrm{K}$, Kriener $\mathrm{S}$ et al. Liver fibrosis in viral hepatitis. noninvasive assessment with acoustic radiation force impulse imaging versus transient elastography. Radiology. 2009;252: 595-604.

19. Sporea I, Sirli RL, Deleanu A et al. What did we learn from the first 3,459 cases of liver stiffness measurement by transient elastography (FibroScan $\left.{ }^{\circledR}\right)$ ? Ultraschall Med. 2011;32:40-5.

20. Lupsor M, Badea R, Stefanescu H et al. Performance of a new elastographic method (ARFI technology) compared to unidimensional transient elastography in the noninvasive assessment of chronic hepatitis C. Preliminary results. J Gastrointestinal Liver Dis. 2009;18:303-310.

21. Castéra L, Vergniol J, Foucher $\mathrm{J}$ et al. Prospective comparison of transient elastography, Fibrotest, APRI, and liver biopsy for the assessment of fibrosis in chronic hepatitis C. Gastroenterology. 2005;128:343-50.

22. Hennes EM, Zeniya M, Czaja AJ et al. Simplified criteria for the diagnosis of autoimmune hepatitis. Hepatology. 2008;48:169-76.

23. Idezuki Y. General rules for recording endoscopic findings of esophagogastric varices (1991). Japanese Society for Portal Hypertension. World J Surg. 1995;19:420-2.

24. Bedossa P, Poynard T. An algorithm for the grading of activity in chronic hepatitis C. The METAVIR Cooperative Study Group. Hepatology. 1996;24:289-93.

25. Kleiner DE, Brunt EM, Van Natta $M$ et al. Design and validation of a histological scoring system for nonalcoholic fatty liver disease. Hepatology. 2005;41:1313-21.

26. Bota S, Herkner H, Sporea I et al. Meta-analysis: ARFI elastography versus transient elastography for the evaluation of liver fibrosis. Liver Int. 2013;33:1138-47.

27. D'Onofrio M, Crosara S, De Robertis R et al. Acoustic radiation force impulse of the liver. World J Gastroenterol. 2013;19:4841-9.

28. Bota S, Sporea I, Sirli R, Popescu A, Gradinaru-Tascau O. How useful are ARFI elastography cut-off values proposed by meta-analysis for predicting the significant fibrosis and compensated liver cirrhosis? Med Ultrason. 2015;17:200-5.

29. Salzl P, Reiberger T, Ferlitsch M et al. Evaluation of portal hypertension and varices by acoustic radiation force impulse imaging of the liver compared to transient elastography and ALT to platelet ratio index. Ultraschall
Med. 2014;35:528-33.

30. Liu H, Fu J, Hong R, Liu L, Li F. Acoustic Radiation Force Impulse Elastography for the Non-Invasive Evaluation of Hepatic Fibrosis in Non-Alcoholic Fatty Liver Disease Patients: A Systematic Review \& Meta-Analysis. PLoS One. 2015;10:e127782.

31. Takahashi H, Ono N, Eguchi Y et al. Evaluation of acoustic radiation force impulse elastography for fibrosis staging of chronic liver disease: a pilot study. Liver Int. 2010;30:53845.

32. Rifai K, Cornberg J, Mederacke I et al. Clinical feasibility of liver elastography by acoustic radiation force impulse imaging (ARFI). Dig Liver Dis. 2011;43:491-7.

33. Sporea I, Bota S, Peck-Radosavljevic M et al Acoustic Radiation Force Impulse elastography for fibrosis evaluation in patients with chronic hepatitis $\mathrm{C}$ : an international multicenter study. Eur J Radiol. 2012;81:4112-8.

34. Yap WW, Kirke R, Yoshida EM, Owen D, Harris AC. Non-invasive assessment of liver fibrosis using ARFI with pathological correlation, a prospective study. Ann Hepatol. 2013;12:608-15.

35. Haque M, Robinson C, Owen D, Yoshida EM, Harris A. Comparison of acoustic radiation force impulse imaging (ARFI) to liver biopsy histologic scores in the evaluation of chronic liver disease: A pilot study. Ann Hepatol. 2010;9:289-93.

36. Takahashi H, Ono N, Eguchi Y et al. Evaluation of acoustic radiation force impulse elastography for fibrosis staging of chronic liver disease: a pilot study. Liver Int. 2010;30:53845.

37. Pfeifer L, Strobel D, Neurath MF, Wildner D. Liver stiffness assessed by acoustic radiation force impulse (ARFI) technology is considerably increased in patients with cholestasis. Ultraschall Med. 2014;35:364-7.

38. Attia D, Pischke S, Negm AA et al. Changes in liver stiffness using acoustic radiation force impulse imaging in patients with obstructive cholestasis and cholangitis. Dig Liver Dis. 2014;46:625-31.

39. Karlas TF, Pfrepper C, Rosendahl J et al. Acoustic radiation force impulse (ARFI) elastography in acute liver failure: necrosis mimics cirrhosis. Z Gastroenterol. 2011;49:443-8.

40. Kuroda H, Kakisaka K, Oikawa $\mathrm{T}$ et al. Liver stiffness measured by acoustic radiation force impulse elastography reflects the severity of liver damage and prognosis in patients with acute liver failure. Hepatol Res. 2015;45: 571-7.

41. Coco B, Oliveri F, Maina AM et al. Transient elastography: 
a new surrogate marker of liver fibrosis influenced by major changes of transaminases. J Viral Hepat. 2007;14:360-9.

42. Sandrin L, Fourquet B, Hasquenoph JM et al. Transient elastography: a new noninvasive method for assessment of hepatic fibrosis. Ultrasound Med Biol. 2003;29:1705-13.

43. Palmeri ML, Wang MH, Rouze NC et al. Noninvasive evaluation of hepatic fibrosis using acoustic radiation force-based shear stiffness in patients with nonalcoholic fatty liver disease. J Hepatol. 2011;55:666-72.

44. Dong DR, Hao MN, Li C et al. Acoustic radiation force impulse elastography, FibroScan ${ }^{\circledR}$, Forns' index and their combination in the assessment of liver fibrosis in patients with chronic hepatitis B, and the impact of inflammatory activity and steatosis on these diagnostic methods. Mol Med Rep. 2015;11:4174-82.

45. Morishita N, Hiramatsu N, Oze T et al. Liver stiffness measurement by acoustic radiation force impulse is useful in predicting the presence of esophageal varices or highrisk esophageal varices among patients with HCV-related cirrhosis. J Gastroenterol. 2014;49:1175-82.

46. Bota S, Sporea I, Sirli R et al. Value of acoustic radiation force impulse elastography for the assessment of ascites syndrome. World J Radiol. 2011; 3: 205-209.

47. Sharma P, Mishra SR, Kumar M, Sharma BC, Sarin SK. Liver and spleen stiffness in patients with extrahepatic portal vein obstruction. Radiology. 2012;263:893-9. 